\title{
Impacto da pandemia da COVID-19 sobre transplantes de córnea
}

\author{
Impact of COVID-19 pandemic on corneal transplantations
}

Carlos Eduardo Ximenes da Cunha' ${ }^{1}$ (i), Santília Tavares de Castro e Silva ${ }^{1}$ (i), Marina Viegas Moura Rezende Ribeiro ${ }^{1}$ (D), Fabiano Timbó Barbosa ${ }^{2}$ (i)

Centro Universitário Tiradentes, Maceió, AL, Brasil ${ }^{2}$ Universidade Federal de Alagoas, Maceió, AL, Brasil.

\section{Cunha CE, Castro e Silva ST, Ribeiro MV Barbosa FT. Impacto da pandemia da COVID-19 sobre transplantes de córnea. Rev Bras Oftalmol. Como citar:}

Cunha CE, Castro e Siva ST, Ribeiro MV, Banbosa FT. Impacto da pandemia da COVID-19 sobre transplantes de cónea. Rev Bras Oftalmol. 2022;81:e0009.

https://doi.org/10.37039/1982.8551.20220009

\section{Descritores:}

COVID-19; Infeções por coronavirus; Transplante de córnea; Pandemias

Keywords:

COVID-19; Coronavirus infections; Corneal transplantation; Pandemics

Recebido: $7 / 4 / 2021$

Aceito: 11/7/2021

Autor correspondente: Carlos Eduardo Ximenes da Cunha Av. Comendador Gustavo Paiva, 5017 Cruz das Almas, Maceió - AL, 57038-000

E-mail: ocaduximenes@gmail.com

Instituição de realização do trabalho: Centro Universitário Tiradentes, Maceió,

$$
\text { AL, Brasil. }
$$

Fonte de auxílio à pesquisa: trabalho não financiado. Conflitos de interesse:
os autores declaram que não há conflitos de interesses.

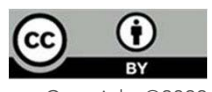

Copyright (C2022

\section{RESUMO}

Objetivo: Tendo em vista que o sistema visual representa uma via de contaminação para o novo Corona vírus, a The Global Alliance of Eye Bank Association (GAEBA) divulgou um guideline preconizando a prevenção de transmissão do vírus entre o doadores de tecido ocular e o pacientes receptores, o que resultou em uma diminuição no número de ceratoplastias e aumento de filas de espera para transplantes de córnea (TC) em todo o mundo. Nesse sentido, o presente trabalho tem por objetivo analisar a situação do transplante de córnea (TC) no estado de Alagoas durante o primeiro ano de pandemia da COVID-19.

Métodos: Trata-se de um estudo analítico, transversal, observacional utilizando dados provenientes de 3 fontes diferentes: Banco de Tecido Ocular Humano de Alagoas (BTO-AL), Registro Brasileiro de Transplantes (RBT) e Agência Nacional de Vigilância Sanitária (ANVISA).

Resultados: Durante os últimos seis anos, foram realizados 284 TCs em Alagoas, segundo dados do Banco de Transplante de Olhos do estado (BTO), já segundo a Registro Brasileiro de Transplantes (RBT) foram realizados 451 TCs. A porcentagem de córneas não oriundas do BTO de Alagoas se mostrou crescente até 2019, porém decresceu em 2020, e no primeiro ano de pandemia, apenas 11,66\% da necessidade anual estimada foi atendida, sendo o período com menor número de TCs realizados entre os seis anos analisados na pesquisa.

Conclusão: $\bigcirc$ estudo demonstra o declínio na doação e realização de transplantes de córnea durante o primeiro ano da pandemia da COVID-19 em Alagoas.

\section{ABSTRACT}

Objective: Considering the visual system is one source of contamination for the new coronavirus, The Global Alliance of Eye Bank Association (GAEBA) released a guideline recommending prevention of virus transmission between eye tissue donors and recipients, which resulted in a reduction of keratoplasties and increase in the waiting lists for corneal transplants worldwide. Hence, this work aims to analyze the situation of corneal transplantation in the state of Alagoas, during the first year of COVID-19 pandemic. Methods: This is an analytical, cross-sectional, observational study using data from 3 different sources: Human Eye Tissue Bank of Alagoas (BTO-AL), Brazilian Transplant Registry (RBT) and National Health Surveillance Agency (ANVISA)

Results: During the past six years, 284 corneal transplants were performed in Alagoas, according to data from the Eye Transplant Bank (BTO) of the state, whereas according to the Brazilian Transplant Registry (RBT) 451 corneal transplants were performed. The percentage of corneas not coming from the BTO of Alagoas increased until 2019 but dropped in 2020. In the first year of the pandemic, only $11.66 \%$ of estimated annual need was met, and this period had the lowest number of corneal transplants performed out of the six years analyzed in the research.

Conclusion: The study demonstrated the decline in donation and performance of corneal transplants during the first year of the COVID-19 pandemic in Alagoas. 


\section{INTRODUÇÃO}

Em março de 2020, a Organização Mundial da Saúde (OMS) decretou pandemia pelo novo coronavírus 2019 (COVID-19), um RNA vírus zoonótico, que circula entre mamíferos por meio de morcegos e dromedários, causando uma doença respiratória aguda com repercussões sistêmicas no organismo infectado. ${ }^{(1)}$ Além da síndrome respiratória aguda, sintomas oculares foram constatados em pacientes com COVID-19, como hiperemia conjuntival, sensação de corpo estranho, olho seco, fotofobia, epífora, dentre outros. ${ }^{(2)}$

Além do mais, o sistema visual também representa uma via de contaminação para o novo coronavírus. Isso porque as células corneanas possuem relativa expressão do receptor ACE-2, componente celular utilizado pelo vírus para infectar as células corporais. ${ }^{(2)}$

Por essa razão, a Global Alliance of Eye Bank Association (GAEBA) uma diretriz preconizando a prevenção de transmissão do vírus entre doadores de tecido ocular e pacientes receptores. ${ }^{\left({ }^{3}\right)}$ As medidas a serem adotadas pelos bancos de olhos incluem a não doação do órgão por indivíduos positivos para COVID-19, suspeitos que vieram a óbito em menos de 14 dias após resolução de sintomas, aqueles sem resultado de teste imunológico e os que tiveram contato com casos suspeitos ou confirmados de COVID-19..$^{(4)}$

Nesse cenário, os bancos de tecido ocular de todo o mundo passaram a restringir a captação de córneas conforme as recomendações de saúde, tendo em vista a possibilidade de contaminação entre doadores infectados e pacientes receptores. ${ }^{(5)}$ Outrossim, a limitação do número de viagens e de doadores efetivos de córnea resultou na redução de ceratoplastias e no aumento de filas de espera para transplantes. ${ }^{(5)}$

O estado de Alagoas apresentou o primeiro caso de COVID-19 no dia $1^{\circ}$ de fevereiro de 2020. Desde então, a Secretaria do Estado da Saúde de Alagoas (SESAU) tem adotado medidas de isolamento social e restrições em serviços de saúde, com a finalidade de diminuir o número de internações pelo vírus da síndrome respiratória aguda grave 2 (SARS-CoV-2) e evitar a superlotação de leitos de terapia intensiva no estado. ${ }^{(6)}$

Mesmo com a adoção de medidas sanitárias, o estado totalizou mais de 3.000 óbitos e mais de 130 mil casos confirmados de COVID-19 em pouco mais de 1 ano de pandemia. ${ }^{(7)}$

Diante dessa situação e da redução mundial das doações de tecido ocular, o presente estudo teve por objetivo analisar a situação do transplante de córnea (TC) no estado de Alagoas durante o primeiro ano de pandemia da COVID-19.

\section{MÉTODOS}

Trata-se de estudo analítico, transversal, observacional, acerca da situação do TC no estado de Alagoas durante o primeiro ano de pandemia da COVID-19. Para tal, foram colhidas informações públicas sobre o TC no estado, referentes ao período entre janeiro de 2015 e dezembro de 2020.

O estudo foi realizado em março de 2021 e utilizou dados provenientes de três fontes diferentes: Banco de Tecido Ocular Humano de Alagoas (BTO-AL), Registro Brasileiro de Transplantes (RBT) e Agência Nacional de Vigilância Sanitária (Anvisa). Os seguintes aspectos foram considerados nesta pesquisa: necessidade anual estimada de TCs; número absoluto de TCs realizados ao ano; número de transplantes por ano no estado com córneas oriundas do Banco de Tecido Ocular (BTO); número de transplantes por ano no estado com córneas não oriundas do BTO; idade em anos dos indivíduos submetidos ao TC e sexo dos pacientes que realizaram o TC. Após a coleta, os dados foram separados e estudados com o uso do aplicativo Microsoft Excel 2016.

Para a discussão, foram utilizados artigos publicados em periódicos indexados nos bancos de dados da Literatura Latino-Americana e do Caribe em Ciências da Saúde (Lilacs), Scientific Electronic Library Online (SciELO) e United States National Library of Medicine (PubMed ${ }^{\circledR}$ ), sendo empregados filtros de idioma, textos na língua portuguesa e inglesa. Os descritores "corneal transplantation", "pandemic" e "COVID-19" foram usados; utilizando AND, foi estabelecida a seguinte relação: "corneal transplantation", "pandemic" AND "COVID-19".

Por se tratar de um estudo realizado com dados públicos disponíveis para a população, essa pesquisa dispensou avaliação por um Comitê de Ética em Pesquisa (CEP).

\section{RESULTADOS}

Este estudo analisou a situação dos TCs no estado de Alagoas nos últimos 6 anos (2015-2020), dando ênfase à evolução deles em 2020, primeiro ano da pandemia do COVID-19.

Durante os 6 anos inclusos neste estudo, foram realizados 284 TCs em Alagoas, segundo dados do BTO-AL, já segundo o RBT, foram realizados 451 TCs. Essa discrepância de dados permite calcular o número de córneas não oriundas do BTO-AL, conforme dados da tabela 1 e da figura 1. 
Tabela 1. Comparação entre o banco de dados do Registro Brasileiro de Transplantes e o do Banco de Tecido Ocular Humano de Alagoas acerca de indivíduos que realizaram transplante de córnea

\begin{tabular}{|cccc}
\hline Ano & $\begin{array}{c}\text { TCs BTO } \\
(\mathbf{n})\end{array}$ & $\begin{array}{c}\text { TCs RBT } \\
(\mathbf{n})\end{array}$ & $\begin{array}{c}\text { Córneas não oriundas do BTO* } \\
(\%)\end{array}$ \\
\hline 2015 & 82 & 86 & 4,65 \\
2016 & 64 & 82 & 20,9 \\
2017 & 74 & 109 & 42,6 \\
2018 & 21 & 79 & 53,2 \\
2019 & 26 & 69 & 62,3 \\
2020 & 17 & 26 & 34,6 \\
\hline
\end{tabular}

Fonte: Banco de Tecido Ocular Humano, Maceió (AL); Registro Brasileiro de Transplantes.

*Percentagem das córneas não oriundas do BTO-AL calculada a partir do número de TCs informado pelo RBT (representa o total de TCs realizados no estado com ou sem córneas oriundas do BTO-AL). TC: transplante de córnea; BTO: Banco de Tecido Ocular; RBT: Registro Brasileiro de Transplantes.

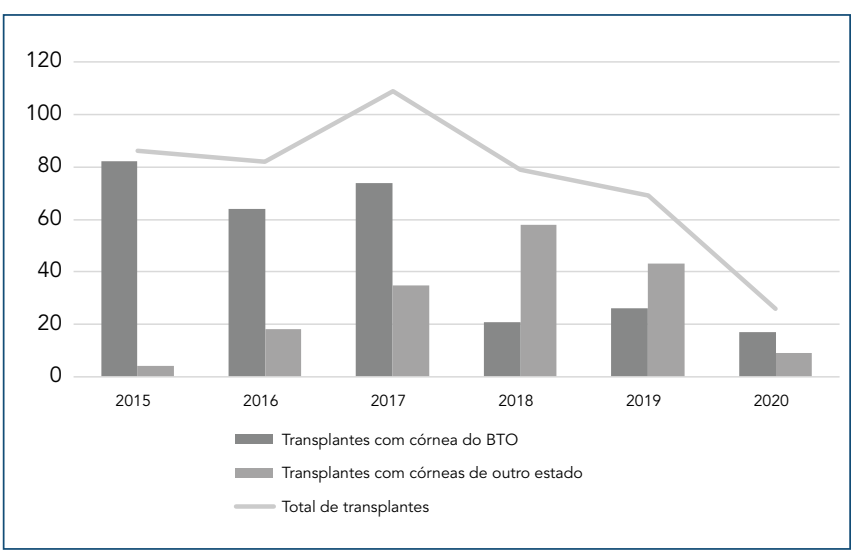

Fonte: Banco de Tecido Ocular Humano, Maceió (AL); Registro Brasileiro de Transplantes. BTO: Banco de Tecido Ocular.

Figura 1. Relação entre transplantes realizados que utilizaram córneas doadas no estado e córneas não oriundas do BTO de Alagoas, considerando o total de transplantes realizados.

A porcentagem de córneas não oriundas do BTO-AL se mostrou crescente até 2019, porém decresceu em 2020, passando de 4,65\%, em 2015, para 20,9\%, em 2016, atingindo pico de $62,3 \%$, em 2019, e decrescendo para 32,3\%, em 2020.

A tabela 2 traz informações de frequência da variável idade, com média de 57,18 anos (desvio-padrão de 21,39 anos) em 2015. A média de idade foi flutuante durante os anos do estudo, tendo seu pico no ano de 2020, com média de idade de 66,55 anos e desvio-padrão de 17,40 anos.

Tabela 2. Idade dos participantes

\begin{tabular}{|lcc|}
\hline Ano & $\mathbf{n}$ & $\begin{array}{c}\text { Idade } \\
\text { anos, média } \pm \text { desvio-padrão }\end{array}$ \\
\hline 2015 & 82 & $57,18 \pm 21,39$ \\
2016 & 62 & $58,82 \pm 20,16$ \\
2017 & 70 & $51,07 \pm 21,29$ \\
2018 & 21 & $56,71 \pm 21,43$ \\
2019 & 19 & $61,95 \pm 20,45$ \\
2020 & 9 & $66,55 \pm 17,40$ \\
\hline Fonte: Banco de Tecido Ocular Humano, Maceió (AL).
\end{tabular}

Os dados da tabela 2 foram fornecidos pelo BTO-AL, porém nem todos os pacientes transplantados tiveram esse dado alimentado. Como mostra a figura 2, 47\% dos pacientes não possuíam o registro de idade. Dentre os pacientes com esse dado, os maiores de 70 anos (29\%) foram os que mais realizaram transplante.

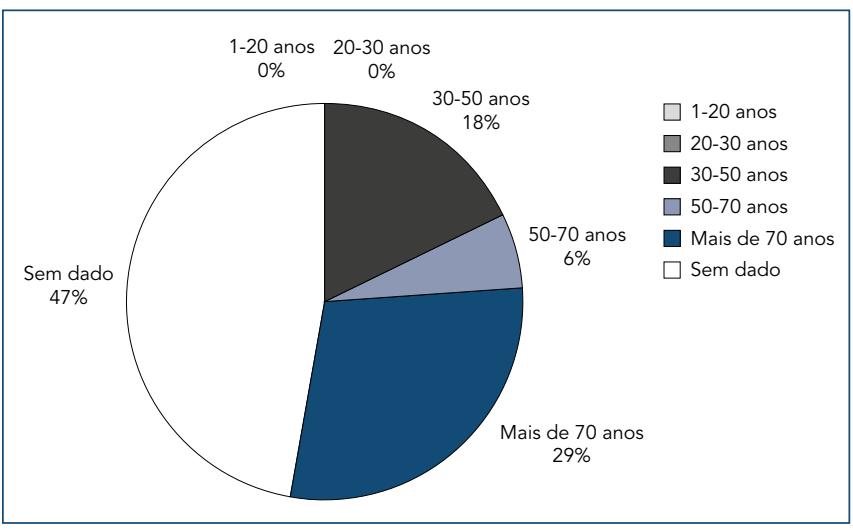

Fonte: Banco de Tecido Ocular Humano, Maceió (AL).

Figura 2. Idade dos indivíduos que realizaram transplante de córnea em 2020.

O número de transplantes no sexo feminino é predominante nos anos do estudo, exceto em 2018. Em 2020, primeiro ano de pandemia, o sexo feminino manteve-se em alta, com $42,3 \%$, e apenas $23 \%$ foram do masculino, sendo o restante dados não fornecidos (Tabela 3).

Tabela 3. Transplante dividido por sexo por ano.

\begin{tabular}{|ccccc|}
\hline Ano & $\begin{array}{c}\text { Transplantes } \\
(\mathbf{n})\end{array}$ & $\begin{array}{c}\text { Dados não fornecidos } \\
(\%)\end{array}$ & $\begin{array}{c}\text { Sexo feminino } \\
(\%)\end{array}$ & $\begin{array}{c}\text { Sexo masculino } \\
(\%)\end{array}$ \\
\hline 2015 & 86 & 4,6 & 51,1 & 44,1 \\
2016 & 82 & 21,9 & 45,1 & 32,9 \\
2017 & 109 & 32,1 & 36,7 & 31,1 \\
2018 & 79 & 73,4 & 11,3 & 15,1 \\
2019 & 69 & 62,3 & 15,9 & 13 \\
2020 & 26 & 34,6 & 42,3 & 23 \\
\hline
\end{tabular}

Conforme dados da tabela 4, a necessidade anual estimada possuía grande variação entre os anos, mas, em 2020, houve a menor necessidade anual estimada (223), enquanto, em 2018, foi registrada a maior (304).

Tabela 4. Necessidade anual e transplantes realizados por ano, número absoluto de transplantes e percentual de efetividade

\begin{tabular}{|cccc|}
\hline Ano & $\begin{array}{c}\text { Necessidade anual estimada } \\
(\mathbf{n})\end{array}$ & $\begin{array}{c}\text { Transplantes } \\
(\mathbf{n})\end{array}$ & $\begin{array}{c}\text { Realizados } \\
(\%)\end{array}$ \\
\hline 2015 & 299 & 86 & 28,7 \\
2016 & 301 & 82 & 27,4 \\
2017 & 302 & 109 & 36 \\
2018 & 304 & 79 & 25,9 \\
2019 & 299 & 69 & 23 \\
2020 & 223 & 26 & 11,66 \\
\hline Fonte: Registro Brasileiro de Transplantes & & \\
\hline
\end{tabular}

Entre os anos de 2019 e 2020, houve queda de 26\% da necessidade anual estimada. Já o número absoluto de 
transplantes diminuiu em 62,4\%. Assim, apenas 11,66\% da necessidade anual estimada foi atendida no ano de 2020, sendo o ano com menor número de TCs realizados entre os 6 anos analisados na pesquisa.

\section{DISCUSSÃO}

Desde que foi anunciada pela OMS, a pandemia da COVID-19 fez com que órgãos de saúde recomendassem medidas restritivas aos hospitais, com a intenção de diminuir chances de transmissão pelo SARS-CoV-2 e melhor direcionar esforços e materiais a pacientes acometidos pela doença. ${ }^{(8,9)}$ Dentre as restrições, as cirurgias eletivas, na qual estão inclusos os TCs, foram impactadas negativamente e tiveram redução importante em seus números. ${ }^{\left({ }^{(0)}\right.}$

Nesse cenário, as ceratoplastias eletivas, mesmo apresentando baixo risco de contaminação por meio do tecido estromal da córnea, foram suspensas, assim como outros procedimentos oftalmológicos não urgentes. ${ }^{(9,10)}$ Segundo a Eye Bank Association of America (EBAA), em julho de 2020 foi observada um redução de $81 \%$ no número de TCs, comparando a média de transplantes realizados anualmente. ${ }^{(10)}$

Em Alagoas, o único BTO do estado, localizado no Hospital Universitário Professor Alberto Antunes (HUPAA), foi remanejado, e uma parte dele foi transformada em unidade para atendimento de casos da COVID-19, assim como em outros locais do mundo. ${ }^{(9)} \mathrm{A}$ associação desse fator, com a restrição de procedimentos eletivos no hospital, pode ter sido responsável pela redução mais expressiva no número absolutos de TCs por ano durante o período analisado - uma queda de aproximadamente $62 \%$ entre 2019 e 2020 . Além disso, a efetividade de realização dos TCs no estado, ou seja, o percentual de transplantes realizados no ano em relação à necessidade anual estimada teve a menor taxa dos últimos 5 anos (cerca de $11 \%)$.

Posta a restrição de cirurgias eletivas, o TC é considerado tratamento definitivo e emergencial nos casos de ceratite e perfuração cornenana. Dessa forma, as ceratoplastias terapêutica e tectônica tenderam a representar a maior parte dos transplantes em alguns locais do mundo. ${ }^{(11,12)}$

Em contraste com a literatura ${ }^{(8)}$, a ceratoplastia óptica continuou sendo a mais realizada em Alagoas, inclusive no primeiro ano de pandemia, no qual foi possível observar que as operações não adiáveis ganharam pouco espaço. Nos últimos 5 anos, a média anual de transplantes terapêuticos e tectônicos foi de 21\% sobre o total de procedimentos realizados. Em 2020, houve aumento de aproximadamente 9\% desse número.
Outro dado que chamou atenção foi a redução no número de procedimentos tanto com córneas oriundas do BTO, como os realizados com córneas vindas de outros estados. A diminuição no número de doadores de córnea foi um fator relacionado diretamente à queda no número de TCs no primeiro ano de pandemia, além de ser resultado das restrições sanitárias para doação de órgãos sólidos e da maior dificuldade de transporte entre diferentes regiões do país. ${ }^{(3,12)}$

Mesmo que ainda não existam estudos comprovando a presença de SARS-CoV-2 em tecidos oculares após a morte, é indicado o rastreio com técnica de reação em cadeia da polimerase via transcriptase reversa (RT-PCR) em doadores sintomáticos em menos de 14 antes do óbito. ${ }^{(13,14)}$ Tendo em vista os problemas com disponibilidade de testes, infraestrutura hospitalar e fechamento parcial de bancos de olhos, houve, no Brasil, diminuição de 44,3\% nos TCs só no primeiro semestre de 2020. ${ }^{\left({ }^{15)}\right.}$

\section{CONCLUSÃO}

Houve declínio na doação e na realização de transplantes de córnea durante o primeiro ano da pandemia da COVID-19 em Alagoas. Essa redução é multifatorial e se deu tanto pela impossibilidade de doação, devido aos novos protocolos, como pela dificuldade de logística e transporte de córneas não oriundas do Banco de Tecido Ocular de Alagoas.

Entretanto, este estudo analisou um único cenário, apenas no estado de Alagoas, durante o primeiro ano de pandemia, sem comparações com outros estados brasileiros. Desse modo, novos estudos, a longo prazo e em outros cenários, são necessários, para analisar o impacto da pandemia sob os transplantes de córnea.

\section{REFERÊNCIAS}

1. Tortorici MA, Veesler D. Structural insights into coronavirus entry. Adv Virus Res. 2019;105:93-116. doi: 10.1016/bs.aivir.2019.08.002

2. Torres BRS, Cunha CEXD, Castro LR, Brito LMP, Ferreira CVO, Ribeiro MVMR. Ocular manifestations of COVID-19: a literature review. Rev Assoc Med Bras (1992). 2020;66(9):1296-300. doi: 10.1590/1806-9282.66.9.1296

3. Gupta PC, Kumar MP, Ram J. COVID-19 pandemic from an ophthalmology point of view. Indian J Med Res. 2020;151(5):411-8. doi: 10.4103/ijmr.IJMR_1369_20

4. Global Alliance of Eye Bank Associations. ALERT UP-DATE: Coronavirus (COVID-2019) and ocular tissue donation. Global Alliance of Eye Bank Associations; 2020 [cited 2021 Jul 5]. Available from: https://www.gaeba. org/2020/alert-coronavirus-2019-ncov-and-ocular-tissue-donation/

5. Ang M, Moriyama A, Colby K, Sutton G, Liang L, Sharma N, et al. Corneal transplantation in the aftermath of the COVID-19 pandemic: an international perspective. $\mathrm{Br}$ J Ophthalmol. 2020;104(11):1477-81. doi: 10.1136/bjophthalmol-2020-317013

6. Torres J. Estatísticas colocam Alagoas como referência nacional no combate à pandemia da Covid-19. Alagoas: Secretaria de Estado da Saúde de Alagoas; 2020 [citado 2021 Jul 5]; Disponível em: https://www. saude.al.gov.br/estatisticas-colocam-alagoas-como-referencia-nacionalno-combate-a-pandemia/ 
7. Torres J. Alagoas tem 132.245 casos da Covid-19 e 3.010 óbitos. Alagoas: Secretaria de Estado da Saúde de Alagoas; 2021 [citado 2021 Jul 5]; Disponível em: https://www.saude.al.gov.br/alagoas-tem-132-245-casosda-covid-19-e-3-010-obitos/

8. Diaz A, Sarac BA, Schoenbrunner AR, Janis JE, Pawlik TM. Elective surgery in the time of COVID-19. Am J Surg. 2020;219(6):900-902. doi: 10.1016/j. amjsurg.2020.04.014

9. Toro M, Choragiewicz T, Posarelli C, Figus M, Rejdak R; European COVID-19 Cataract Group (\#EUROCOVCAT). Early Impact of COVID-19 Outbreak on the Availability of Cornea Donors: Warnings and Recommendations. Clin Ophthalmol. 2020;14:2879-2882. doi: 10.2147/OPTH.S260960

10. AlMutlak M, Li JY, Bin Helayel H, Fairaq R. Future of corneal donation and transplantation: insights from the COVID-19 pandemic. Cornea. 2021;40(3):274-6. doi: 10.1097/ICO.0000000000002538
11. Chaurasia S, Sharma N, Das S. COVID-19 and eye banking. Indian J Ophthalmol. 2020;68(6):1215-6. doi: 10.4103/ijo.IJO_1033_20

12. Roy A, Das S, Chaurasia S, Fernandes M, Murthy S. Corneal transplantation and eye banking practices during COVID-19-related lockdown period in India from a network of tertiary eye care centers. Indian J Ophthalmol. 2020;68(11):2368-71. doi: 10.4103/ijo.IJO_2258_20

13. Sawant OB, Singh S, Wright RE 3rd, Jones KM, Titus MS, Dennis E, et al. Prevalence of SARS-CoV-2 in human post-mortem ocular tissues. Ocul Surf. 2021;19:322-329. doi: 10.1016/j.jtos.2020.11.002

14. Ballouz D, Mian SI. Eye banking in the coronavirus disease 2019 era. Curr Opin Ophthalmol. 2020;31(5):389-95. doi: 10.1097/ICU.0000000000000684

15. Associação Brasileira de Transplante de Órgãos (ABTO). Dados numéricos da doação de órgãos e transplantes realizados por estado e instituição no período: janeiro/junho - 2020. São Paulo: ABTO; 2020 [citado 2021 mar 26]. 22 p. Disponível em: https://site.abto.org.br/publicacao/ano-xxvi-no-2/ 